\title{
Butterfly Learning: An Approach for Appropriating Complexity in Science Education for Young Children
}

\author{
Jiangbo $\mathrm{Hu}^{1}$, Camilla Gordon ${ }^{2}$ \\ ${ }^{1}$ Zhejiang Normal University, China \\ ${ }^{2}$ Macquarie University, Australia
}

\begin{abstract}
This paper focuses on one element of the results of a study which has explored the socially constructed and family centred science education program - Young Starwatcher. This program is characterised with its informal and collaborative structure that involves a variety of families and their preschool and early primary school children who experienced a series of activities for developing the understanding in certain astronomical concepts in an easy and digestible way. The challenge has been to understand the appropriation of learning dealing with the complexity of astronomical concepts within a group of "just plain folks" who lack astronomy knowledge. This study sets to explore the actual processes that make learning informally but collaboratively a powerful tool for children and parents. While visual imagery, storytelling and other educational activities were used in the program to facilitate the participants' understanding in the abstract astronomy concepts, "Butterfly learning" was initiated from the interviews with the parents and the observations on the learning activities as a method for promoting participants' motivation and understanding. "Butterfly learning" is a metaphor that represents learners gaining knowledge and understanding in a pattern of "a small step in each time of learning" informally, just like a butterfly sourcing nutrition in natural environment bit by bit randomly. This approach allows the participants to acquire information repeatedly at their own pace, which preceded their development of understanding in complexity as a joyful journey. Butterfly learning appears to play a pivotal role in both children's behaviour and learning method.
\end{abstract}

\section{Introduction}

This study is based on a science education program named Young Starwatcher that was designed for a group of parents and their preschool and early primary school children's (24) learning on the topics of astronomy and the Earth. Participants in the Starwatcher programme meet monthly at the Nepean Observatory to learn Earth and Planetary science for three years. The appropriation of such a complex and often abstract science is daunting to the people who lack knowledge in the area. Special procedures have been employed within the program to deconstruct scientific understandings. "Butterfly learning" was one of these. The term "butterfly learning' is used as a metaphor to demonstrate the specific process of the program that characterised with "a small step at each time of learning" where the participants appropriated the complexity of astronomy concepts.

Butterflies are creatures of great beauty and initiative, drawn by instinct through some internal mechanism to seek out the necessities for life in whatever scenario they find themselves. They are as varied as humans are individual, always evolving in metamorphic cycles. They have proved to be as vital to the flowers from which they sup nectar as the flowers are to them as a source of nutrition. This source of food is found deep within the cone of petals and ensures that pollen from stamens touches the wings and feet of the butterfly and is transferred to other flowers. Thus, fertilisation is achieved, and the cycle of plant life goes on. Butterflies also supplement their diet with mud, especially wet clay which supplies them with nutrients not available in nectar. How they know to do this is somewhat of a mystery, but they appear to be patterned genetically.

Just as this extracting of nutrient does not appear to have ever been exactly measured, so butterfly learning is unable to be measured or assessed, even in individual children since it is the repetition and revisiting in concrete learning situations that builds the understanding. It differs from museum learning and most types of school learning which seeks effectiveness in learning environments when information is transferred from adults to children. In this research setting, the children and the parents are discovering together, neither with more initial knowledge dominance until a form of understanding is achieved, and then it is shared. Vygotsky [8] sheds light on the learning that is being observed:

\footnotetext{
"In working its slow way upward, an everyday concept clears a path for the scientific concept in its downward development. It creates a series of structures necessary for the evolution of a concept's more primitive, elementary aspects, which give it body and vitality. Scientific concepts in turn supply structures for the upward
} 


\begin{abstract}
development of the child's spontaneous concepts toward consciousness and deliberate use." (p. 109)
\end{abstract}

Vygotsky explicitly acknowledged the role of deconstruction of learning process in the formation of children's everyday concepts to scientific concepts. In the Young Starwatcher program, the development of scientific concepts is in a slowly unpacking pattern. Participants in the program have relied on the hands-on activities structured in a long journey to facilitate and support understandings. The significance of this study over the past three years is that it provided an informal learning opportunity that is both complex and abstract relying on the continual building of 'snippets' of understanding. Subsequent learning and knowledge construction results from placing small understandings into a bigger picture, a picture containing metaphysical acknowledgements and the puzzle of abstract physics.

\section{Complexity of the Science program}

It is important here to classify what is meant by complexity in this science program. In this study, this term refers to a series of astronomy concepts that describe the formation of our environment or the "history of the Earth". A 'nebula' is, to put it simply and etymologically, a cloud of gas and dusts which results from the death or explosion of a star. From time to time stars reach the end of their long lives and return to the form from which they began. Some do this in dramatic fashion, in a huge explosion called a supernova when a star exhausts its fuel and ends its life, but most just fizzle away as a geriatric body of gas and dust. Our solar system began when a cloud of this 'geriatric' dust and gas bumped into a cloud from a supernova. After billions of years, the resulting star, our sun exploded into nuclear life and cast off the excrement gas and dust into the eight planets. All the small parts of "big things" on the Earth were made of stardust, billions of years ago.

A nebula therefore becomes one of the focus knowledge to deconstruct for understanding in the history of the Earth. This cloud of gas and dust is so immense that close to $97 \%$ of it is absorbed by the star (the sun) and only 3\% is left for the eight planets. Taking into consideration that four of the planets are very much bigger than Earth, it stands to reason that this 'nebula' must have been massively larger than comprehension allows. Figure 1 shows a montage of Planetary Nebulae observed with the Hubble Space Telescope.

This abstract, absent and unbelievably gigantic 'thing' is the core of the complex knowledge that needs to be understood since almost all astronomical knowledge requires understanding that star formation begins with gas and dust. The terms 'gas' and 'dust' are frequently used and have distinct meanings in the commonplace. In astronomical terms, they take on new meaning as untamed, fiercely hot, powerful and pervasive throughout the solar system and galaxy. The effects of dust entering our atmosphere is commonly seen in the night sky as 'shooting stars'. Such comet and meteor showers events are frequently celebrated in the media - the Leonids for example.

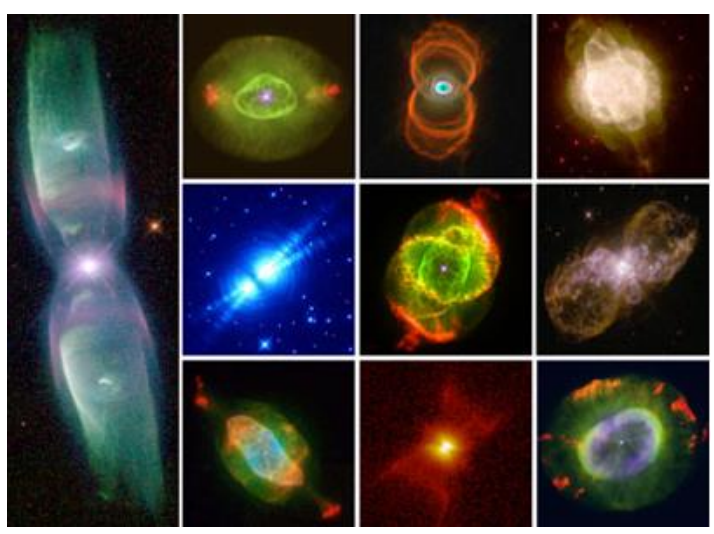

Figure 1. A montage of Planetary Nebulae

The challenge here is to understand the process that 'just plain folks' (families) go through to appropriate such complexity. Vygotsky [9] clarifies a notion of a representation between the abstract scientific concepts of the adult and the preconceptual representations of children which is the "pseudoconcept". For Vygotsky, 'pseudo-concepts' were crucial in enabling children to join in episodes of instruction with teachers.

"The apparent similarity of the pseudoconcept and the scientific concept enables a teacher to act toward the child as if he/she were further advanced in understanding the concept than actually is the case. The teacher interprets the child's actions in terms of a higher level of development but in so doing reveals to the child the path toward cultural expertise. Some misunderstanding may occur, but in the context of instruction, it can be productive because it reveals to the teacher and the child the underlying structure of the concepts and creates conditions for a more deliberate and reflective grasp of concepts." [9] (p.119)

Young children have natural interest in space and astronomic phenomena. They developed some pseudo-concepts about the Moon, the Earth, and other cosmic bodies through daily observations. 
Though these concepts may be inaccurate, they would accept the scientific knowledge with careful designed science program. Kampeza [10] reported a study of 76 children aged 5 to 6 years. Their findings indicated that, with elementary astronomy concepts, many of the children understood the shape of the Earth to be spherical but had relatively poor knowledge of the day/night cycle and the movement of the planets. However, despite astronomy concepts being abstract and counter-intuitive, they reported that with the use of appropriate artefacts and acting out the movement of astronomical objects, young children can appropriate elementary astronomy that appears to be too difficult for their age.

Hannust and Kikas [14] conducted a study in Estonia with 113 children aged 5 to 7 years to examine their pre-existing knowledge of the Earth. This was assessed using questions and drawing tasks. An experimental group (half of the children) was given an instructional astronomy intervention similar to the curriculum requirement for 7-year-old children and all participants were assessed again. This study set out to specifically address a finding of previous research by Vosniadou and Brewer [13] which indicated that "specific entrenched beliefs must be addressed in order for children to accept scientific explanations" (p. 93). Hence the children's everyday knowledge, together with inconsistencies and entrenched beliefs, was discussed and reviewed with the children to assist them to make conscious connections between their everyday understandings and accurate scientific information. Results indicated that most of the children's knowledge was fragmented and not consistent with scientific accuracy before instruction. Post instruction results showed that although the majority of the children had not achieved scientifically accurate models, there was an improvement in the post-test. This result confirmed that even young children can understand factual information about the Earth. Results also suggested that children were not inhibited by 'entrenched' ideas as suggested by Vosniadou and Brewer [13] but were able to able to embrace further learning. "Therefore, early instruction may be recommended to satisfy children's curiosity and to introduce the topics related to the Earth and gravity" [14] (p. 102).

The previous studies demonstrated young children's capabilities in understanding abstract astronomy concepts. However, these studies undertaken with well-designed intervention program in formal educational settings. The current study differs from these studies by deconstructing the concepts through a series of learning activities organized informally in a non-educational setting. The children and their parents participated in the activities spontaneously based on their interest and life routines. This study sake to explore whether such a learning pattern, named "butterfly learning" is effective for supporting children to gain understanding in complex astronomy concepts.

\section{Qualitative Inquiry Research}

This study is structured by qualitative inquiry research method. The goal of qualitative inquiry is not to produce just knowledge of the social/scientific world but to engage in knowledge making as a human activity. Because qualitative research concerns itself with the meanings and personal experience of individuals, groups and sub-cultures. The nature of qualitative research involves the reflection on 'truth' through a series of subjective accounts. This has been attempted through observation and documentation, through interview and discussion, and through hermeneutic reflection in collaboration with participants. In this scenario, the researchers have become self-reflexive, playing a role which theoretically sees validity embracing subjectivity, while at the same time being trustworthy enough to be relied upon [12].

While phenomenological reflection seeks to perceive the meanings of human experiences, deconstructing the 'realness of learning' in astronomy science is more difficult. The hermeneutic interview reflection that uncovered the focus of this paper had a conversational structure, exploring the range and varieties of experiential material that had been collected for the phenomenon under study, and interpreting the aspects of meaning that was associated with this phenomenon. It had its orientations in sense making and the interpretation of experiential meanings. It was during one of these sessions that the term 'butterfly learning' was used to describe the learning, which was just touching the periphery of the subject, the 'that' of knowledge, the building of experience.

Dewey [6], in exploring the essence of experience, postulates that everything is what it is experienced. Hence, if we want to describe anything truly, we need to tell what it is experienced as being. Though this experience may be of the vaguest sort, it is this vagueness, this confusion that makes the experience, and which makes the experience absolutely unique. Whatever clarity is gained, or truth is experienced it must grow out of some element of the experience and this experience is an unequivocal, personal reality [6][4].

All experiences are irrevocably rooted in the power of culture and tradition, in both moral and scientific areas. When a person is involved in an experience, it is not some grandiose remote affair that is cast like a net around a succession of fleeting experiences: "... things are what they are experienced to be, and that everything is something". [6] (p.247). As mentioned above, one of Vygotsky's [9] premises is that accumulating knowledge through experience supports a steady 
growth of scientific reasoning, which in its turn influences the development of spontaneous thinking. This support base of experiential, everyday representations of things forms the platform from which scientific concepts are formed.

The sociocultural perspective suggests that learning is a process of appropriating tools for thinking', that are made available by social agents who initially act as interpreters and guides in the individual's cultural apprenticeship [11]. It is not just that the child learns from others in social contexts and during social exchange, but rather that the actual means of social interaction (language, gesture) are appropriated by the individual (internalised and transformed) to form the intramental tools for thinking, problem-solving, remembering, and so on [1].

In this study, the interactions among the parents, children and the staff of Nepean Observatory created a learning context where the participants enhanced their understanding in the abstract scientific concepts in their individual way in the step-by-step learning journey. This study was set to explore whether parents and children could develop their understanding in the complexity of astronomical concepts through the approach of "butterfly learning"; and how did they view such a learning approach.

\section{Participants and the Learning Program}

About 24 Families and their preschool aged children attended the Young Starwatcher program. They each month joined in an activity specifically to learn astronomy in an easy, digestible way. Lave's [7] ethnographic studies of learning and everyday cognition focus on what she calls 'just plain folks'. These families were "just plain folks", aspiring to learn particular things, and doing this automatically as an apprenticeship of engaging in a task. Their understanding of this complex science was based on regular activity nights where everyone could play with and act on the materials provided. Activities at Yong Starwatchers were usually designated according to a theme which was, in turn, determined by the sky - such as the craters visible on the halfmoon; the constellations such as Scorpio and Orion which are visible at different times of the year; and the passing by of the International Space Station (ISS) or the Hubble Space Telescope (HST).

Into these timely events, other activities were woven - Earth Science for instance, addressed the question that if dust is the most prolific substance in the Universe, what exactly is dust? Provided with huge sandstone rocks, hammers and goggles, children and adults converted the whole rock into sand, and then to finer and finer particles until dust was obtained. Along the way they were introduced to ways of using the sand and dust - layering it in a jar, as it was originally formed into rocks millions of years ago, or adding clay, crushed shale and compost, in an attempt to make soil. While the adults might be able to visualise the possibilities and the end-product, children often were experiencing each step as an end in itself and moving on to the next without reference to any larger picture. This was to be expected and this was built into the programming. However, it is this very 'step-by-step' glimpses of understanding that have provided wonderings about the distance each child/adult had travelled cognitively and metacognitively. Planning how to approach a learning opportunity, monitoring comprehension, and evaluating progress towards completion was often not considered until the particular activity had been acted upon for many months. The participants gradually built up their knowledge and understanding in the scientific concepts with the step-by-step exploration collaboratively.

\section{Data collection and analysis}

The data was collected through two sources that include: a) record on the interactions among the parents and their children; b) open interview to parents for investigating their opinions on the Young Starwatcher program. The main researcher recorded the parent-child interactions in Nepean Observatory with video camera and made field notes. The interviews were arranged with negotiations with the parents regarding when and where the interviews being undertaken. Each family was interviewed once and five families who attended the program regularly were interviewed for three time throughout the tree-year learning journey. The main researcher had built up close relationships with these families during the years, which facilitated the openness of the interviews.

The recorded video clips were viewed for several times by the researchers and the dialogues between the parents and the children's relating to the astronomical learnings were identified and transcribed. The transcribed dialogues were analysed based on the speakers' language containing deductive thinking that connect two or more than two scientific ideas. These dialogues demonstrate the participants' development in scientific concepts. The parent interviews were transcribed thoroughly. The interview data was refined according to whether it reflected the participants' scientific understanding or their attitudes regarding to the learning pattern throughout the three years. The conclusions were drawn taking into the consideration of the findings from the two sources. 


\section{Findings}

\subsection{Participants' understanding in astronomical concepts}

The records on parent-child interactions show that both the parents and the children gained good understanding in a series of astronomical concepts, though the extent may vary. For the families who attended the learning activities more regularly, the parents and the children tended to have more conversations, and showed sound understanding in the astronomical concepts. In a conversation with a six-year-old and her mother, there are indications of the way children interweave everyday understandings into a complex phenomenon based on newly learnt scientific knowledge.

Dialogue 1:

Mother: Why do we keep going around
the sun?
Child: We're going in orbit around the
sun
Mother: And what do you think about the
moon...
What does the moon do?
Child: ... it moves, and the reason we see
it in the night-time, and it looks like it's
staying in the same place, is that we're
moving and it's moving.
Mother: That's exactly right, so it orbits
like us?
Child: Yes.
Mother: So it would be very unlikely for
us to see it full all the time wouldn't it,
because it's going round us like we're
going round the sun.
Child: It's like the sun's the boss!
Mother: Yeah, you got that right, the sun
is the boss! Child: And then the moons
are ... and then the planets are the
servants and then the moons are the
slaves!

This next dialogue occurred between a child and a father. The child is 4 years old and was discussing how he felt about Starwatcher program and what he learnt about volcanos. In this dialogue, the boy showed keen interest in the program because it brought him new things. He tried to state the "new things" that he had learnt about dust and gas, the Earth, fire, volcanoes and rocks. Though his statement was not completely accurate, he described the basic relationships among the natural phenomena, involving his deductive thinking.
Dialogue 2:

Child: I liked hearing new things like new things are really good, like just learning well ...

Father: Learning about the world? Child: Maybe, maybe about the planets too.

Father: What did you learn? What do you think you learned at Starwatchers that you wouldn't have known if you hadn't been to Starwatchers?

Child: Nearly everything!

Father: That'll make Camilla (the researcher) happy saying that. But what do you think you learnt? When we went there last time, was there anything that you learnt at the end of that day that you didn't know already?

Child: I didn't know about just the whole world joining up from dust and gas! When rocks hit Earth, fires can ignite plus volcanoes, because volcanoes are popular around here and they're all put out now. And they might just happen up again, because there might be just this little block around the volcano and this rock might just hit it right in.

In an interview, a mother of a seven-year-old boy reported the boy's understanding of gravity when he had an encounter with gravity. According to the mother's description, this seven-year-old child seemed to transfer his scientific knowledge into daily life and applied the scientific concept of gravity to interpret his encounter of falling towards ground in this way:

He was climbing a tree and his jumper got caught on a branch, leaving him suspended, fortunately close to the ground. Once he realised he was caught he yelled; 'Look Mum, gravity has got me!'

When asked what the things in night sky are, several children could apply knowledge of the existence of a nebula in the night sky that they learnt through the learning experiences into their everyday experiences. For example, a four-year-old child showed deductive reasoning in his drawing presence of nebula in the night-time sky. 


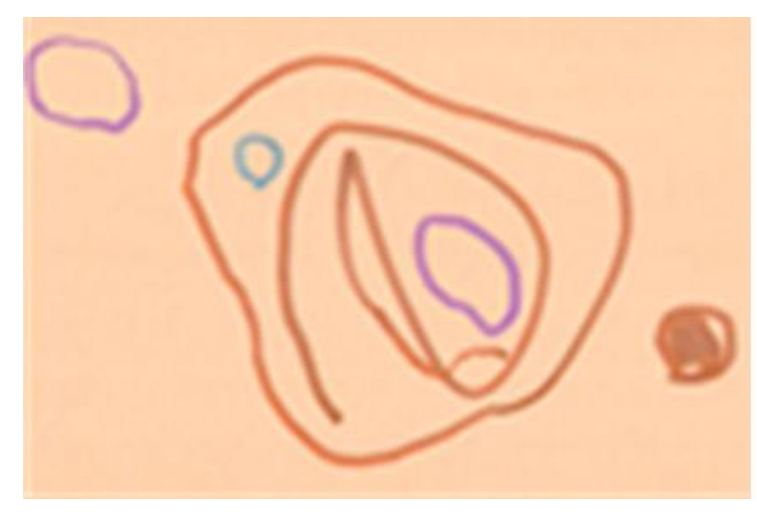

Figure 2. The existence of nebula in the night sky

This child used shape and colour to signify the presence of a nebula between two stars, indicating his understanding in the existence of nebula though he might not see a nebula in the sky. Figure 2 displayed the child's drawing.

\subsection{Participants' attitudes toward the learning pattern - butterfly learning}

The Young Starwatcher program is characterised with its collaborative, informal, and step-by-step learning approach - butterfly learning. The interview data shows that most parents held positive attitudes towards this learning pattern. Parents stated their opinions from different perspectives. The following quotes from parent interviews represent their typical ideas about butterfly learning.

- The perspective from children's learning interest

Successful learning occurs if your child is happy learning. It's not someone who's just scored 10/10 in a test; it's someone who loves learning and all the different approaches to learning - practical or rote. All the different approaches, if they've got a smile on their face, then that's successful learning. If they can remember and file away info, they've got it. Children take you where they want you to go.

- The perspective from a person and of a person

I found children and adults
returning to activities that they
enjoyed or were interested in
repeatedly. In one instance I had a
boy stay with me throughout almost
the entire session, fascinated with the
concept of building a mountain of
sedimentary rocks from different types
of breads, grains, peas etc

\begin{abstract}
(compressed to imitate the action of weight building up on top). His siblings, both younger and older travelled around all the displays like most other children. This boy was so interested that he stayed to see "what we would make" - until we cut through the sedimentary mountain to see the layers. Most other children came and went or visited the display only once.
\end{abstract}

- The perspective from comparison with other learning patterns

In a structured learning situation, he would have been moved on with his group, and probably spent the rest of the time thinking about the sediment mountain and wondering what it was developing into. He probably would not have gained much from the other activities.

\section{Discussion}

This study explored the efficiency of butterfly learning for supporting young children's understanding in complex astronomy concepts. The process of gaining the understanding involves the participants' individual development from everyday concept to scientific concept. The participants went through their discrete "small steps" at their own pace and in their own contexts.

A child returns home from school saying 'I learnt about frogs today'. This statement would appear to denote that his learning was substantial, that he had indeed appropriated the how, why, when and where of frog knowledge. The time frame - today - would suggest otherwise. In the initial stages of understanding about a frog, a child would be introduced to the word "frog" and through actions, songs and visuals, become aware that a frog is a green or brown animal that jumps, spends time in the water and variously croaks or says galumph! Obviously, there is a great deal more to learn (and some of that early learning will be proven to be incorrect). However, the child is running with the information he has, much the same as Harlow Shapely and Heber Curtis did in the early part of this century [2]. Each of these men insisted on his own view of the size of the universe; Shapely saw our cosmos as a "flattened disc of stars" (p.32), essentially one galaxy, the Milky Way. Curtis's cosmos was much, much larger - filled with disc shaped galaxies, millions or even billions of light years wide. Seife [2] states the uselessness of the argument because neither man had the tools or 
opportunities to prove or disprove his assertions. They were simply debating the knowledge of the day. It wasn't long before a young man called Edwin Hubble proved that not only was the universe massive beyond belief, and filled with galaxies, he also discovered that the universe was expanding and may well expand forever [2].

Both the child and the scientists, Shapely and Curtis, used the knowledge they had to hand. This is entirely appropriate since all knowledge is disseminated from current understandings and with complex science those understandings are staged according to culture and opportunity [8]. When we use butterfly learning to appropriate complexity, we 'taste' or 'extract snippets' of knowledge and fit them into existing paradigms.

It is inferred then that butterfly learning is the beginnings of inculcation of a learning procedure; that it constitutes a gradual process of information reality, and that it is possible in specially designed learning environments where opportunities are able to be repeated and revisited. It is further inferred that when complex learning is allowed be a prolonged step-by-step process and artefacts of the learning are collected along the way, without constraint of structure or assessment, then children/adults will come to a point of understanding from which they can move forward.

Ryle [5] suggests that ..... "Learning how or improving ability is not like learning that or acquiring information. Truths can be imparted, procedures can only be inculcated, and while inculcation is a gradual process, imparting is relatively sudden. It makes sense to ask at what moment someone became apprised of a truth, but not to ask at what moment someone acquired a skill" (p.58)

This type of learning is necessarily embedded in metaphysics, in the ontological understandings of engaged practices, in blending understandings into established frameworks where vision and understanding are rooted in the commonplace. If play is defined as an act of freedom then play appears to be at the centre of butterfly learning. It is the essence of play in learning that allows the mind to touch, to reach into the unknown and discover something new. It is best conceived as a process where ideas are drawn-out, tested and re-learnt [3][15]. Children explore play as non-obligatory sense making, where this form of meaning creation is not "bound" to the physical world, and yet it is not without structure. Through play the world needs not have fixed meanings. That which in the "open sensemaking" is a film container now suddenly is a rocket; a cardboard role becomes a telescope, or a musical instrument. Children used their imagination to "play their meanings" out, from which they gained understanding and practiced their understanding. Butterfly learning in this study served as a springboard to provide children with plenty of opportunities for learning abstract scientific concepts through fun and repeat play, and this play was at their own pace. This learning pattern was welcomed by the participants because it suits children's nature.

\section{Conclusion}

This study has implications for the science education community, given the lack of direct research into the processes of knowledge construction in informal contexts, and how groups of people of different ages and abilities appropriate complex understandings. Informal learning is always viewed as an undesirable learning approach for learning complex knowledge. Yet, this research demonstrates the potential of an informal learning context for both adults and children to gain understanding in complex scientific concepts with pleasant experiences. As a parent reported:

The opportunity to spend as much or as little time as one wishes to, on any one topic, allows us to absorb information while our interest is held (which is when we absorb it best), and to move on when we are bored or satisfied (when we have received as much information as we can digest comfortably). The opportunity to return for more when the appetite returns makes the whole experience very pleasurable and memorable - another fact that enhances recollection and learning. With no-one insisting we "digest a fixed amount of information - no more no less" we can satisfy our own personal needs, and enjoy the experience, guilt free and pressure free.

There is value for everyone in this form of butterfly learning. It is learning with enjoyment, and no negative impacts on self-image or self-esteem. The ideas on butterfly learning presented here indicate that early science learning is yet to be structured within such a potential. The complexity of scientific concepts involved in the learning could be well addressed with this approach

\section{Reference}

[1] B. B. Bernstein, Pedagogy, symbolic control and identity: Theory, research critique (Rev. Ed.), Rowman \& Littlefield Publishers, Lanham, Maryland, 2000.

[2] C. Seife, "Cosmology. Galaxy maps support theory that the universe is flying to pieces", Science, 2003, 302(5646), pp. 762-763. 
[3] H. Marit Holm, "How children make meaning through drawing and play", Visual Communication, 2008, 7(1), pp.77-96.

[4] E, J. Kipton, "John Dewey's philosophy of spirit. (critical essay)" The Pluralist, Spring, 2013, 8(1), pp. 129(9).

[5] G. Ryle, (1949). The concept of mind. London: Hutchinson's University Library, 1949.

[6] J. Dewey, The philosophy of John Dewey, Putnam Sons, New York, 1973.

[7] J. Lave, Cognition in practice, mind, mathematics and culture in everyday life, Cambridge University Press, New York, 1988

[8] L. S. Vygotsky, Mind in society: The development of higher psychological processes, Harvard University Press, Cambridge, Mass, US, 1978.

[9] L. S.Vygotsky, The development of scientific concepts in childhood. In E. Hanfmann \& G. Vakar (Eds.), Thought and language: Studies in communication. (pp. 82-118), MIT Press. Cambrige, Mass, US, 1962.

[10] M. Kampeza, "Preschool children's ideas about the Earth as a cosmic body and the day/night cycle ideas", Journal of Science Education, 2006, 7(2), pp. 119-122.

[11] M. Mccaslin, H. L. Burross, "Research on individual differences within a sociocultural perspective: Coregulation and adaptive learning", Teachers College Record, 2011, 113(2), 325-349.

[12] N, K. Shanhid, "Qualitative research method Phenomenology" Asian Social Science, 2014 10(21), pp. 224-233. doi: 10.5539/ass.v10n21p298

[13] S. Vosniadou, W.F. Brewer, "Mental models of the earth: a study of conceptual change in childhood", Cognitive Psychology, 1992, 24(4), pp. 535-585.

[14] T. Hannust, E. Kikas, "Children's knowledge of astronomy and its change in the course of learning", Early Childhood Research Quarterly, 2007, 22(1), pp. 89-104.

[15] T. R. Godlstein, P. Bloom, "Characterizing characters: How children make sense of realistic acting", Cognitive Development, 2015, pp. 34, 39-51. 